\title{
Funciones Ejecutivas y Rendimiento Académico en Educación Primaria de la Costa Colombiana
}

\section{Porto M.F. ${ }^{1}$, Puerta-Morales, Laura ${ }^{2}$, Gelves-Ospina, Me- lissa $^{3}$, Urrego-Betancourt, Yaneth ${ }^{4}$}

${ }^{1}$ Departamento de Psicología, Universidad de la Costa, Barranquilla

${ }^{2}$ Departamento de Psicología, Deutsche Schule, Barranquilla

${ }^{3}$ Departamento de Psicología, Universidad del Norte, Barranquilla

${ }^{4}$ Universidad Piloto De Colombia, Bogotá

\section{Colombia}

Correspondencia: María Fernanda Porto. Department of Psychology, Universidad de la Costa, Cl. $58 N^{\circ} 55$ 66, Barranquilla, Atlántico, Colombia. E-mail: mporto3@cuc.edu.co 


\section{Resumen}

Introducción. Las Funciones Ejecutivas (FE) son un conjunto de funciones supramodales que favorecen a las habilidades cognitivas, emocionales y sociales. En la primera infancia las FE influyen en el rendimiento de las habiliadades académicas. La presente investigación determinó la relación entre las funciones ejecutivas y el rendimiento académico en un contexto educativo.

Método. Se realizó un estudio correlacional donde participaron 195 estudiantes entre 6 y 12 años de edad, seleccionados al azar de los grados de básica primaria (de $1^{\circ}$ a $5^{\circ}$ ). Las funciones ejecutivas medidas fueron: fluidez verbal (fonológica y semántica), atención selectiva, flexibilidad cognitiva, planificación e inhibición; para lo cual, se empleó la Evaluación Neuropsicológica de las funciones ejecutivas en niños (ENFEN) y el rendimiento académico se evaluó a partir del promedio académico acumulativo en el año.

Resultados. De acuerdo con el análisis de correlación de Spearman, los resultados arrojados señalan que existe una relación estadísticamente significativa entre rendimiento académico y los componentes de las funciones ejecutivas: fluidez fonológica $(p=.01)$ y flexibilidad cognitiva $(p=.01)$. Por otro lado, la regresión logística arrojó que la fluidez semántica y la inhibición son factores predictivos para el rendimiento académico en un $76.4 \%$.

Discusión y conclusiones. Se sugiere que las funciones ejecutivas se asocian y a su vez predicen el rendimiento academico en estudiantes de básica primaria, en especial si este es bajo.

Palabras clave: funciones ejecutivas, fluidez verbal, inhibición, rendimiento académico, básica primaria. 


\begin{abstract}
Introduction. The Executive Functions (EF) are a set of supramodal functions that favor cognitive, emotional and social skills. In early childhood, EFs influence the performance of academic skills. The present research determined the relationship between executive functions and academic performance in an educational context.
\end{abstract}

Method. A correlational study was conducted involving 195 students between 6 and 12 years of age, randomly selected from primary grades (from 1 to 5). The executive functions measured were: verbal fluency (phonological and semantic), selective attention, cognitive flexibility, planning and inhibition; for which, the Neuropsychological Evaluation of the executive functions in children (ENFEN) was used and the academic performance was evaluated from the cumulative academic average in the year.

Results. According to Spearman's correlation analysis, the results show that there is a statistically significant relationship between academic performance and the components of executive functions: phonological fluency $(p=.01)$ and cognitive flexibility $(p=.01)$. On the other hand, the logistic regression showed that semantic fluency and inhibition are predictive factors for academic performance in $76.4 \%$.

Discussion or Conclusion. Which suggests that executive functions are associated and in turn predict academic performance in elementary school students, especially if this is low.

Keywords: executive functions, verbal fluency, inhibition, academic achievement, elementary school. 


\section{Introducción}

Las funciones ejecutivas (FE), término acuñado por Lezak (1982), hace referencia a un conjunto de capacidades cognitivas que involucran el control atencional, la memoria de trabajo, inhibición, fluidez verbal, velocidad de procesamiento de información, flexibilidad cognitiva, establecimiento de objetivos, control de impulsos, empatía y toma de decisiones (Anderson, 2008; Portellano, 2005; Stelzer y Cervigni, 2011; Tobar, 2014).

De igual modo, se reconoce la corteza pre-frontal como base neuroanatómica de dichos procesos necesarios para el ajuste del comportamiento social, la regulación emocional y la planificación de comportamientos cognitivamente complejos (Martínez y Manoiloff, 2010; Tirapu-Ustárroz y Luna-Lario, 2008; Yang y Raine, 2009; Wiebe et al., 2011). Es relevante entender que su formación y consolidación tienen un tiempo establecido en la ontogénesis; y su debida estimulación en la etapa escolar, puede ayudar a un mejor desempeño académico (Anderson, Jacobs y Anderson, 2008).

De manera particular, el rendimiento académico es un factor determinante para la permanencia de los estudiantes en la escuela (Torres, Acevedo y Gallo, 2015). El Instituto de Estadística de la Organización de las Naciones Unidas para la Educación, la Ciencia y la Cultura UNESCO-IEU (2012) revela que aproximadamente 32.2 millones de estudiantes de educación primaria repitieron el grado en el que se encontraban y 31.2 millones abandonaron la escuela. En la Costa del Caribe Colombiano, se ha encontrado que la deserción escolar es una problemática que influye negativamente en el desarrollo escolar, cognitivo, familiar y social de los niños (Erazo, 2012). En particular, Barranquilla tiene una tasa de deserción del 4.15\% desde el 2008 (Ministerio de Educación Nacional [MEN], 2011).

En este sentido, existen programas que evalúan internacionalmente este desempeño, tales como el Programa para la Evaluación Internacional de los Alumnos (PISA), y la Organización para la Cooperación y el Desarrollo Económicos (OECD, 2014), que destacan el bajo rendimiento de los estudiantes colombianos representado por un puntaje promedio de 376 para matemáticas, 403 en lectura y 399 en ciencias, los cuales están por debajo de la media internacional, y lo ubican en el puesto 62 de 65 países evaluados (OECD, 2014). Tiramonti (2014) expresa que Colombia y Perú son los países de Latinoamérica que presentan tasas rela- 
tivamente bajas de escolarización y, al mismo tiempo, no logran buenos resultados en las pruebas.

Estudios en neurociencias abren campo a la intersección entre el estudio del cerebro y el aprendizaje. Tsujimoto et al. (2004), afirman que durante diferentes actividades relacionadas con la memoria de trabajo se activan áreas similares tanto en niños como en adultos. Desde el punto de vista educativo, existen dos posturas para el estudio de las FE. La primera reconoce que la capacidad de autorregulación le permite al individuo obtener el logro o éxito académico; y la segunda, postula que el aprendizaje en el contexto educativo estimula a su vez, el desarrollo de las FE (Garner, 2009). Sin embargo, existe un punto de cohesión entre ellas, donde se entiende que las funciones ejecutivas cumplen un rol importante en el proceso de enseñanza-aprendizaje, y en las áreas de conocimiento como el cálculo, la resolución de problemas matemáticos y la lecto-escritura (Toll et al., 2012; Van der Ven et al., 2013).

De esta manera, el colegio es el escenario donde los niños desarrollan su potencial, aprenden conceptos y comportamientos que son modelados por docentes, pares, y la cultura en la que están inmerso (Ortiz, 2013). El rendimiento académico se entiende como la valoración o registro numérico que una institución educativa realiza sobre el desempeño específico de un estudiante, puede ser clasificado en tres niveles: alto, medio y bajo (Puerta, 2015). Dicho desempeño es altamente valorado en ciencias matemáticas y de lengua castellana (Valle et al., 2015). Sin embargo, depende de múltiples variables, como son las físicas, cognitivas, familiares, y sociales (Algozzine y Algozzine, 2009; Lassen, Steele y Sailor, 2006; Rudasill, Gallagher y White, 2010; Stelzer y Cervigni, 2011).

Diversas investigaciones mencionan la relación entre las funciones ejecutivas y el rendimiento académico. Se ha estudiado el efecto de la edad y escolaridad en el aprendizaje, la inteligencia, las habilidades pre-académicas y la cultura (Garner, 2009; Lan, Legare et al., 2011; Rahbari y Vaillancourt, 2015; Roebers y Jäger, 2014; Shaul y Schwartz, 2014; Suchodoletz et al., 2013). Por lo cual, han señalado la necesidad de estudiar la relación bidireccional de las funciones ejecutivas y el rendimiento académico (Titz y Karbach, 2013) aunque este vinculo puede ser deficiente en población con desventajas socioeconómicas (Deer et al., 2020; Peng y Kievit, 2020). Y a pesar del grado de motivación, el rendimiento en flexibilidad cognitiva, la memoria operativa y la inhibición del estudiante (Nieto-Márquez et al., 2021). 
Ahora bien, con respecto a las dificultades en matemáticas, lenguaje y otras áreas, puede verse mediado por un déficit en la realización de actividades de manera planificada y organizada, o incluso el bajo control atencional (De Lima et al., 2011; Toll, Van der Ven et al., 2011). Por lo cual, Suárez-Riveiro et al. (2019) sugieren que a un mayor rendimiento en atención e inhibición mejora el promedio académico global. Sin embargo, esto podría depender según Grenell y Carlson (2021) de si la enseñanza esta basada en hechos o aspectos conceptuales.

Lan et al. (2011) compararon el rendimiento académico (lenguaje y matemáticas), y las funciones ejecutivas (memoria de trabajo, inhibición y control atencional) en estudiantes Chinos y Norteamericanos; donde las diferencias culturales entre los grupos evaluados, fue explicada por el ambiente escolar y las prácticas de enseñanzas inmersas en cada contexto. Además, Scope et al. (2010) afirmaron que hay relación entre la inatención, hiperactividad, e impulsividad, la memoria de trabajo y el control inhibitorio en niños de 8 a 9 años. Lo cual, podría relacionarse por la influencia de contextos menos favorecidos para el desarrollo de las FE (Martins y Gotuzo, 2015).

\section{Objetivos}

Por todo lo anterior, este estudio tuvo como objetivo determinar la relación existente entre las siguientes funciones ejecutivas (inhibición, planificación, control atencional, flexibilidad cognitiva y fluidez verbal), y el rendimiento académico de estudiantes de básica primaria de la ciudad de Barranquilla.

\section{Método}

\section{Participantes}

La población de estudiantes pertenecian al colegio Distrital Libertador Simón Bolívar (I.E.D. L.S.B.), situado en la ciudad de Barranquilla y vinculado a un nivel socioeconómico bajo (Estrato socioeconómico 1 y 2). La muestra fue seleccionada a través de un muestreo de tipo probabilístico, ya que los participantes fueron escogidos al azar con una heterogeneidad del 50\%, 5\% de margen de error y 95\% de nivel de confianza. Se escogieron 200 estudiantes, de los cuales se eliminaron 5 participantes por ausentismo, quedando un total de 195 sujetos. Se seleccionaron al azar 40 estudiantes para cada grado de básica primaria $\left(1^{\circ}, 2^{\circ}, 3^{\circ}, 4^{\circ}\right.$ y $5^{\circ}$ ). Con respecto a la representación por sexo en cada grado, primero tenia un $50 \%$ para 
hombres y mujeres, en segundo grado un $57.5 \%$ para hombres y $42.5 \%$ para mujeres, en tercer grado $42.5 \%$ para hombre y $57.5 \%$ para mujeres, en cuarto grado $57.5 \%$ para hombres y $42.5 \%$ para mujeres. Finalmente, quinto grado tuvo un $47.5 \%$ para hombres y $52.5 \%$ para mujeres. Con respecto a la edad estuvo en un rango de 6 a 12 años con un promedio de 8.70 $(D E: 1.73)$

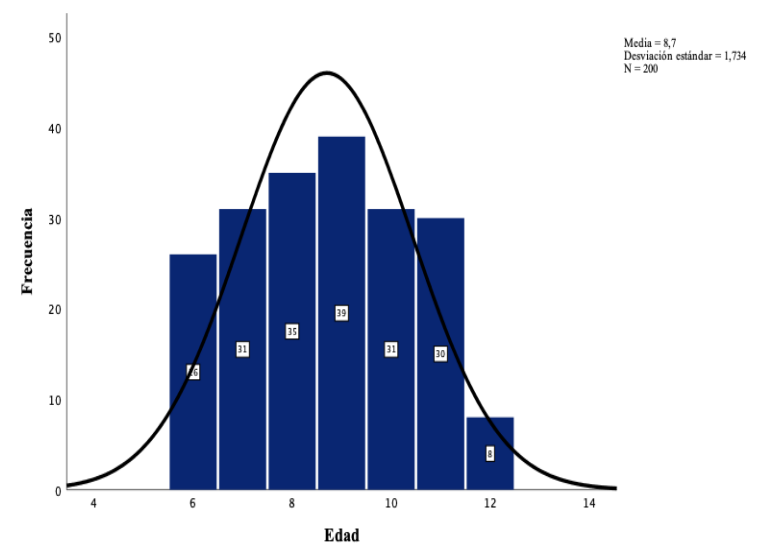

Figura 1. Distribución por edad.
Figura 2. Distribución por sexo.

Los criterios de inclusión para la muestra fueron: cursar básica primaria y tener entre 6 y 12 años de edad. Como criterio de exclusión, se estableció no presentar antecedentes de enfermedad neurológica, trastornos del espectro autista, trastornos psiquiátricos o del neurodesarrollo, discapacidad intelectual, déficit sensorial, motor o problemas graves de comprensión de lenguaje, evidenciado mediante entrevista semi-estructurada dirigida a padres

\section{Instrumentos}

Para la evaluación de las FE se utilizó la Evaluación Neuropsicológica de las Funciones Ejecutivas en Niños (ENFEN) de Portellano et al. (2009), el cual mide el nivel de madurez y el rendimiento cognitivo en actividades relacionadas con las funciones ejecutivas en niños a través de los siguientes cuatro test: fluidez verbal, construcción de senderos, construcción de anillas y resistencia a la interferencia. La evaluación psicométrica recoge 6 índices neuropsicológicos en decatipos que permite la medición de: fluidez fonológica, fluidez semántica, atención selectiva, flexibilidad cognitiva, planificación e inhibición. Igualmente, el ENFEN incluye seis decatipos que establece un perfil de las funciones ejecutivas de un (1) muy bajo a diez (10) muy alto. El análisis factorial realizado sugeridos por Portellano et al. (2009) del manual ENFEN concluyen un factor único. Esto se debió a que, debido a las carac- 
terísticas del sistema ejecutivo prefrontal, se comporta como un sistema (Navarro Soria et al., 2019).

Para medir la variable de rendimiento escolar, se utilizó el promedio académico acumulado, con una puntuación de 1 a 100, categorizados en los siguientes rangos: Entre 1 y 59 (insuficiente), entre 60 a 79 (aceptable), entre 80 a 94 (sobresaliente) y por último, entre 95 a 100 (excelente). Estas medidas a partir de medidas estandarizadas internacionales podrían clasificarse 1-79 bajo, 80-94 medio y 95 a 100 alto (Puerta, 2015).

\section{Procedimiento}

La investigación se llevó a cabo mediante una evaluación individual. En cada evaluación se administró el ENFEN en el mismo orden que sugieren los autores y siguiendo los parámetros de aplicación. La duración promedio de cada evaluación individual fue de 35.2 minutos para los participantes de $1^{\circ}$ a $3^{\circ}$ grado y 19.5 minutos para los estudiantes de $4^{\circ}$ a $5^{\circ}$ grado. La evaluación se realizó durante el horario escolar. Para la variable de rendimiento académico, las directivas de la institución, con la autorización previa de los padres, suministraron reportes detallados del promedio acumulado de los estudiantes.

\section{Consideraciones éticas.}

Todos los procesos investigativos estuvieron guiados bajo los las normas éticas establecidas para la investigación en seres humanos de acuerdo a los estatutos del Colegio Colombiano De Psicólogos, COLPSIC: Ley 1090 del 6 de Septiembre de 2006. De esta forma, durante el desarrollo de la investigación, los padres firmaron el consentimiento informado y se socializó la naturaleza del proceso y los derechos que serían garantizados. El equipo de investigación siguió las normas de aplicación, corrección e interpretación conforme a lo establecido por los autores en el respectivo manual del ENFEN

\section{Análisis de datos}

Para los análisis estadísticos se utilizó el programa SPSS Versión 20 (Statistical Package for the Social Sciences) donde se analizaron de manera inicial las características sociodemográficas mediante distribución de frecuencias relativas (porcentajes) para las variables de genero, grado escolar y edad. Se describieron las variables de estudio de rendimiento académico promedio y Funciones ejecutivas utilizando medidas de tendencia central y dispersión. 
Por medio de la prueba de Spearman, se establece el coeficiente de correlación entre las variables de Rendimiento acdémico promedio y las FE (Fluidez fonológica, Fluidez semántica, Atención selectiva, Flexibilidad cognitiva, Planeación e Inhibición). Para interpretar la correlación se utilizaron los tamaños de efectos sugeridos por Cohen (1986), en donde valores < 0.10: $\sin$ efecto; 0.10 a 0.30 : efecto pequeño; 0.31 a 0.50 : efecto moderado; > 0.51 : efecto grande.

Para la medición de los factores de FE asociados al Rendimiento académico se realizó una regresión logística para analizar el efecto que ejercen las variables independientes continuas (Funciones ejecutivas) sobre las probabilidades que caracterizan la variable dependiente (rendimiento académico). Se obtuvieron en el proceso los coeficientes del modelo, sus errores estándares y el estadístico de wald que permitieron la comparación de modelos en cada paso.

\section{Resultados}

Se evaluaron 195 estudiantes, cuyas edades oscilaban entre los 6 y 12 años, con una mayor proporción de hombres que mujeres. En la tabla 1 se describe la distribución de frecuencias con respecto a las variables sociodemográficas como edad, sexo (varón/mujer), y grado escolar (primero, segundo, tercero, cuarto y quinto).

La Figura 3 muestra el promedio del rendimiento académico y la valoración por frecuencias que obtuvieron los estudiantes. El puntaje más bajo para la medición de rendimiento académico fue 52, que corresponde a la categoría cualitativa de insuficiente y la más alta 92, que corresponde a una categoría de sobresaliente. 


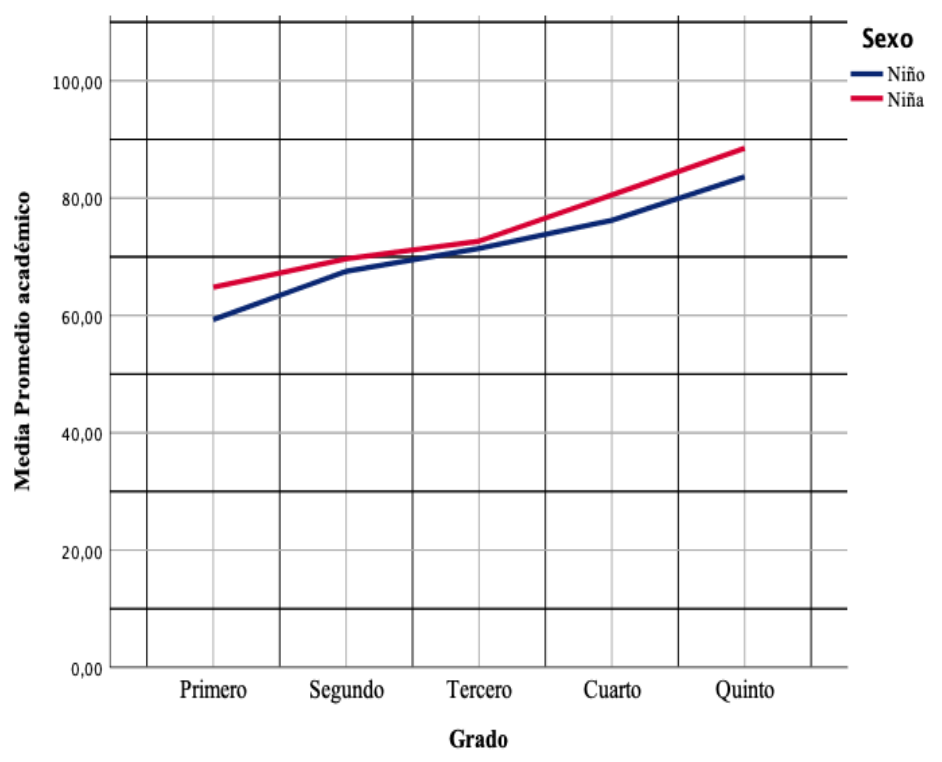

Figura 3. Distribución de promedio académico.

En la Tabla 1, presenta el resumen de las medidas de tendencia central con respecto a las variables analizadas: fluidez verbal (fonológica y semántica), atención selectiva, flexibilidad cognitiva, planificación e inhibición. De acuerdo a las puntuaciones medias en relación con los percentiles para la muestra estudiada, las variables de fluidez verbal, flexibilidad cognitiva, planificación e inhibición se encontraron por encima del segundo percentil (25-50), en contraste con el indicador de atención selectiva, que se ubicó en el primer percentil (0-25).

Con respecto a la asimetría de las curvas para las diferentes funciones ejecutivas, se evidencia dispersión en los datos obtenidos. Sin embargo, con las variables de fluidez fonológica, fluidez semántica, atención selectiva e inhibición, muestran unas curvas placticúrticas evidenciando poca concentración en la media, de acuerdo a la curtosis. Para flexibilidad cognitiva y planificación muestran una mayor concentración de la media con curvas leptocúrticas.

Con respecto a la variable promedio académico, se observó que la mayoría de los estudiantes evaluados se encontraron en la categoría de aceptable 72.98. Con respecto a la dispersión, el rendimiento académico se muestra distribuido a los dos lados de la media, pero con tendencia hacia los promedios académicos más altos, evidenciado en una curva mesocúrtica. 
Tabla 1. Descriptivos de las Funciones ejecutivas y el promedio académico.

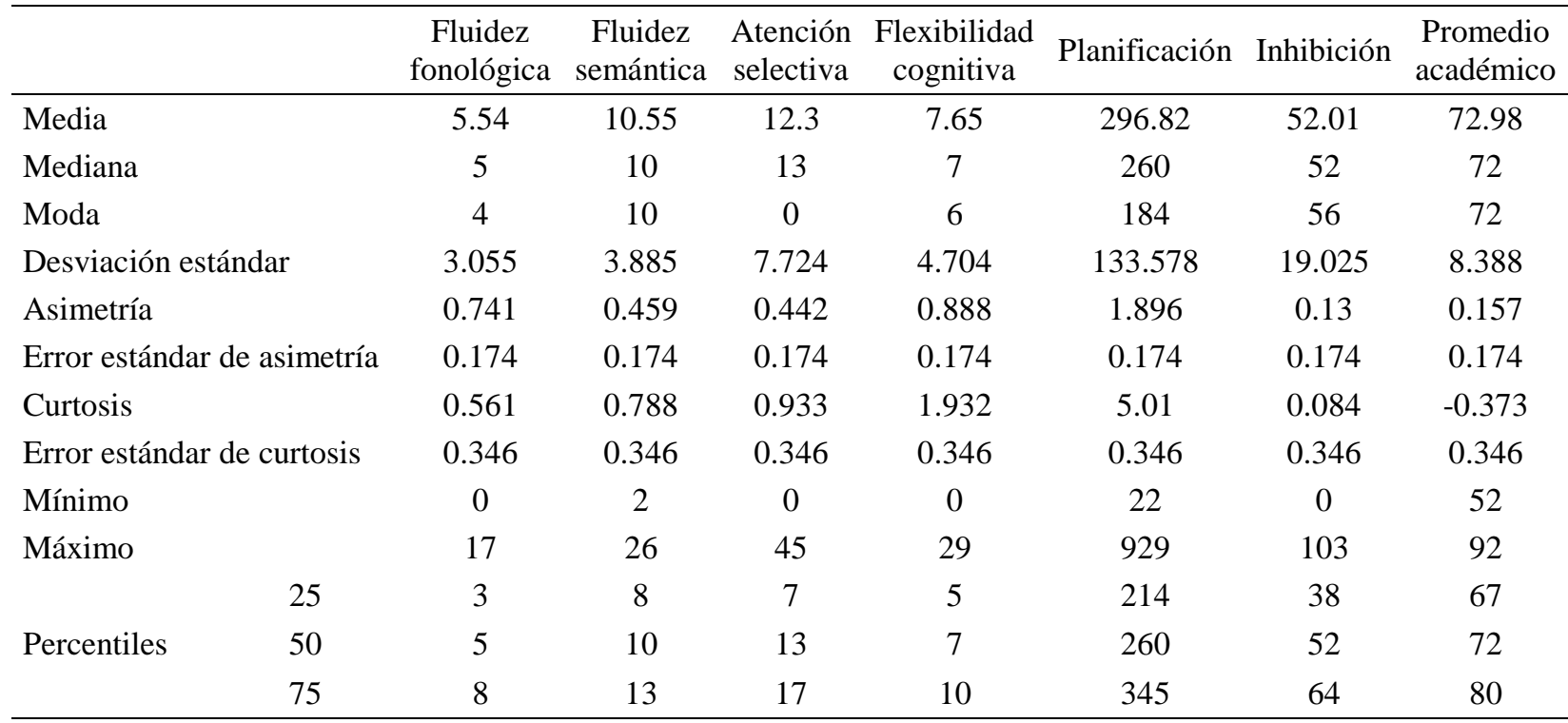

La tabla 2 muestra los resultados arrojados por la $\mathrm{R}$ de Spearman muestran la relación entre las FE y rendimiento académico. El valor obtenido de q (Cohen,1986) evidencia un tamaño de efecto grande para fluidez fonológica (.611) y flexibilidad cognitiva (.607), un efecto pequeño para fluidez semantica (.231) e inihibición (.210) y finalmente, para las variables de planificación (.109) y atención selectiva (.104) un efecto pequeño dentro del límite inferior del rango para este tamaño de efecto.

Tabla 2. Análisis de correlación (Rho de Spearman)

\begin{tabular}{lcccccc}
\hline & $\begin{array}{c}\text { Fluidez } \\
\text { fonológica }\end{array}$ & $\begin{array}{c}\text { Fluidez } \\
\text { semántica }\end{array}$ & $\begin{array}{c}\text { Atención } \\
\text { selectiva }\end{array}$ & $\begin{array}{c}\text { Flexibilidad } \\
\text { cognitiva }\end{array}$ & Planificación & Inhibición \\
\hline $\begin{array}{l}\text { Promedio } \\
\text { académico }\end{array}$ & $.611^{* *}$ & $.231^{*}$ & .104 & $.607 * *$ & .109 & $.210^{*}$ \\
\hline
\end{tabular}

**. La correlación es significativa en el nivel 0,01 (bilateral).

*. La correlación es significativa en el nivel 0,05 (bilateral).

Finalmente, se procedió a determinar el factor predictivo de las funciones ejecutivas sobre la variable rendimiento académico, tal como se observa en la tabla 3. 
Tabla 3. Resultados derivados de la regresión logística binaria para la probabilidad de presentar alto rendimiento académico.

\begin{tabular}{|c|c|c|c|c|c|}
\hline & & \multicolumn{2}{|c|}{ Promedio Académico } & \multicolumn{2}{|c|}{ Porcentaje correcto } \\
\hline & & Alto & Bajo & & \\
\hline \multirow{6}{*}{ Promedio Académico } & Alto & 3 & 45 & & \\
\hline & Bajo & 1 & 146 & & \\
\hline & & $B$ & $E T$ & Wald & $G l$ \\
\hline & $\begin{array}{l}\text { dez } \\
\text { atica }\end{array}$ & .113 & .048 & 5.537 & 1 \\
\hline & ición & -.023 & .009 & 6.289 & 1 \\
\hline & tante & 1.216 & .664 & 3.354 & 1 \\
\hline
\end{tabular}

Nota: $\mathrm{B}=$ coeficiente; E.T. = Error Estándar; Gl= Grados de Libertad.

A través de la regresión lógistica realizada, los resultados arrojan que el modelo logró predecir el $76.4 \%$ de los casos, a través de los factores predictivos de fluidez semántica e inhibición, con valores de significancia de Wald $>0.05$. De acuerdo a lo anterior, se se puede estimar que la fluidez semántica y la inhibición son factores predictivos para el rendimiento académico.

\section{Discusión y Conclusiones}

Esta investigación tuvo como objetivo central correlacionar las funciones ejecutivas y el rendimiento académico en el ámbito escolar. Siendo la escuela un lugar de interacción donde se fortalecen las habilidades cognitivas y sociales, en un ambiente propicio para el desarrollo integral de los niños y niñas. Por lo cual, es importante evaluar las funciones ejecutivas dado que involucran un conjunto de habilidades que permiten al estudiante dar respuesta a las exigencias académicas. A partir de los percentiles de la población estudiada se evidencia un rendimiento medio para las funciones ejecutivas de fluidez verbal, flexibilidad cognitiva, planificación e inhibición y un rendimiento bajo para el indicador de atención selectiva. Fernandez-Castillo y Gutiérrez (2009) afirman que puntajes bajos en la atención podría repercutir en un rendimiento inferior a lo esperado, tal como se evidencio en el promedio académico de la población estudio, con un puntaje cualitativamente aceptable o promedio bajo. 
Al establecer las relaciones entre las variables de estudio, con respecto a la fluidez verbal, se encontró un efecto grande tanto para el aspecto fonológico y efecto pequeño para el semántico con respecto el rendimiento académico. Lo anterior, confirma lo encontrado por Marino, Acosta y Zorza (2011) quienes reportan mejor rendimiento de fluidez verbal a mayor velocidad evocativa con uso de anticipación y monitorio. Ahora bien, en el entorno educativo las estrategias usadas en las diferentes asignaturas concertadas para la primaria por el Ministerio de educación (Ministerio de Educación Nacional, 1984), fortalecen las funciones ejecutivas y el contenido de tipo verbal, permitiendo que se adquieran en un mayor nivel en la fluidez y en la capacidad de recurrir a ellos de forma eficiente.

Asimismo, al analizar la relación entre flexibilidad cognitiva y rendimeinto académico, se encontró que existe efecto grande, lo cual coincide con los hallazgos de Vandenbroucke et al. (2017) los cuales afirman que la flexibilidad cognitiva muestra pequeñas mejoras en el rendimiento académico de los niños de primer grado.

De acuerdo con la medida de inhibición, aunque se encontró una relación débil con respecto al rendimiento académico; diversos autores sostienen que los procesos inhibitorios son fundamentales para el desempeño académico y el éxito académico del niño (Aydmune et al., 2016). Lo cual, es diferenciador en está investigación, ya que el tipo de población y el estrato socioeconómico vulnerable al cual pertenecen reveló un impacto significativo en el rendimiento cognitivo (Aran-Filippetti, 2011; Deer et al., 2020; Peng y Kievit, 2020) y en las habilidades académicas reflejadas en un promedio general de rendimiento académino aceptable para Colombia y bajo según los estándares internalizacionales. Asimismo, al analizar la medida de atención selectiva y planificación se encontró efecto estadísticamente bajo dentro de los límites inferiores de la muestra estudiada, tal como lo reporta Reyes et al. (2015), lo cual puede ser debido a la vulnerabilidad sociodemográficas particulares de la muestra estudiada. Esta variable se debe seguir teniendo en cuenta en próximos estudios.

El análisis de regresión lineal lógistica, evidenció que las variables predictoras en un 76.4\% para el rendimiento académico son fluidez semántica e inhibición. La fluidez semántica se explica desde la velocidad en el procesamiento de información, lo cual tiene una importante relación con la capacidad para aprender y las habilidades de aprovechamiento escolar; ya que la fluidez semántica es aquella tarea donde se pide generar palabras pertenecientes a una categoría (ej.: animales, frutas, ropa, transportes, verduras, etc.), otorgándose un tiempo 
límite para ejecutar la tarea (Ramírez et al., 2005). La inhibición por su parte se relaciona con la capacidad de autoregulación, que influye directamente con la capacidad de aprendizaje, explicada por Del Valle y Urquijo (2015), quienes explican estás variables neurocognitivas predicen en cierta medida el rendimiento académico, específicamente encontraron asociación entre el uso de estrategias semánticas de codificación, la capacidad de aprendizaje y el desempeño académico de los estudiantes.

La importancia de establecer un marco de referencia para el rendimiento cognitivo de los estudiantes de básica primaria en el contexto colombiano radica en la detección temprana de dificultades en el área escolar (Gutiérrez-Martínez y Ramos, 2014); así como en identificar aquellas variables asociadas a las FE que pueden a largo plazo impactar en las pruebas de estado. Igualmente, nuestros resultados favorecen y fomentan a la intervención temprana en FE, que beneficien a los estudiantes en la toma de decisiones y los planes a futuro, mejorando de manera importante el rendimiento académico.

Dentro de las limitaciones del estudio se pueden resaltar la necesidad de contar con la participación de instituciones educativas con estudiantes de diferentes niveles socioeconómicos, para una mayor representatividad de la muestra, así mismo aumentar el tamaño muestral representativo para el país. Kishiyama et al. (2014) afirman que el estrato socioeconómico influye el rendimiento del niño(a) con respecto a las FE, ya que algunas condiciones de crianza, estrés, falta de acceso a estimulación cognitiva, materiales y experiencias, no aportan al rendimiento cognitivo de los escolares. Se espera en proximos estudios implementar guías para la estimulación de las funciones ejecutivas, que permiten minimizar el impacto de las condiciones de vulnerabilidad socio-económica, que conllevan a las conductas sociales disruptivas, el ausentismo, la deserción y la pérdida del año escolar. Se sugiere que esta investigación pueda ser replicada en otras regiones del país, tanto es escuelas públicas como privadas, para tener un panorama completo del rendimiento de las funciones ejecutivas y su relación con el promedio académico. Así mismo, que se pueda establecer una estandarización Colombiana de la bateria ENFEN para ampliar sus usos.

En conclusión, este artículo sugiere que existe una relación significativa entre fluidez fonológica, flexibilidad cognitiva y rendimiento académico. A pesar de esto, las variables que podrían convertirse en un marcador cognitivo para predecir el estado de las habilidades aca- 
démicas en población de estrato socioeconómico vulnerable son la fluidez semántica e inhibición.

\section{Referencias}

Algozzine, B. y Algozzine, K.M. (2009). Facilitating academic achievement through school wide positive behavior support. In W. Sailor, G. Dunlap, G. Sugai y R. H. Horner (Eds.), Handbook of positive behavior support (pp. 521-550). New York: Springer.

Anderson, V., Jacobs, R., y Anderson, P. (2008). Executive function and frontal lobes: A lifespan perspective. New York: Francis y Taylor Group.

Anderson, P. J. (2008). Towards a developmental model of executive function. En V. Anderson, R. Jacobs y P. J. Anderson (Eds.), Executive functions and the frontal /lobes: A lifespan perspective (pp. 3-22). Nueva York: Psychology Press

Arán-Filippetti, V. (2011). Fluidez verbal según tipo de tarea, intervalo de tiempo y estrato socioeconómico, en niños escolarizados. Anales de psicología, 27(3), 816-826.

Aydmune, Y., Zamara, E., Introzzi, E., Richard’s, M. (2016). Relaciones entre la inhibición comportamental, inhibición perceptual y el test stroop, en niños en edad escolar. Anuario de proyectos e informes de Becarios de investigación, 13, 1382-1392.

Cohen, J. A. C. O. B. (1986). Statistical approaches to suicidal risk factor analysis. Annals of the New York Academy of Sciences, 487, 34-41.

De Lima, R., Salgado, C., y Ciasca, S., (2011). Attentional Performance and Executive Functions in Children with Learning Difficulties. Psicologia: Reflexão e Crítica, 24(4), 685-691.

Deer, L. K., Hastings, P. D., y Hostinar, C. E. (2020). The role of childhood executive function in explaining income disparities in long-term academic achievement. Child development, 91(5), e1046-e1063. https://doi.org/10.1111/cdev.13383

Del Valle, M, y Urquijo, S. (2015). Relaciones de las estrategias de codificación mnésica y la capacidad de aprendizaje con el desempeño académico de estudiantes universitarios. Psicología Educativa, 21, 2737.

Erazo, O. (2012). El rendimiento académico, un fenómeno de múltiples relaciones y complejidades. Revista vanguardia psicológica clínica teórica y práctica, 2(2), 144-173.

Fernández-Castillo, A., \& Rojas, M. E. G. (2009). Atención selectiva, ansiedad, sintomatología depresiva y rendimiento académico en adolescentes. Electronic journal of research in educational psychology, 7(1), 4976.

García, T., González-Castro, P., Rodríguez, C., Cueli, M., Álvarez, D., y Álvarez, L. (2014). Alteraciones del funcionamiento ejecutivo en el trastorno por déficit de atención con hiperactividad y sus subtipos. Psicología Educativa, 20, 23-32.

Garner, K. (2009). Conceptualizing the Relations between Executive Functions and Self-Regulated Learning. The Journal of Psychology, 143(4), 405-426. 
Gutiérrez-Martínez, F., y Ramos, M. (2014). La memoria operativa como capacidad predictora del rendimiento escolar. Estudio de adaptación de una medida de memoria operativa para niños y adolescentes. Psicología Educativa, 20, 1-10.

Grenell, A., y Carlson, S. M. (2021). Individual differences in executive function and learning: The role of knowledge type and conflict with prior knowledge. Journal of experimental child psychology, 206, 105079. https://doi.org/10.1016/j.jecp.2020.105079

Instituto de estadística de la UNESCO (UIS). (2012). Compendio mundial de la educación: Oportunidades perdidas; El impacto de la repetición y de la salida prematura de la escuela.

Kishiyama, M., Boyce, T., Jimenez, A., Perry, L., y Knight, R. (2014). Socioeconomic Disparities Affect Prefrontal Function in Children. Journal of Cognitive Neuroscience. 21(6), 1106-1115.

Lan, X., Legare, C., Cameron., Li, S., Morrison, P. (2011). Investigating the links between the subcomponents of executive function and academic achievement: A cross-cultural analysis of Chinese and American preschoolers. Journal of Experimental Child Psychology, 108, 677-692. doi:10.1016/j.jecp.2010.11.001

Lassen, S. R., Steele, M. M. y Sailor, W. (2006). The relationship of school-wide positive behavior support to academic achievement in an urban middle school. Psychology in the Schools, 43, 701-712.

Lezak, M.D. (1982). The problem of assesing executive functions. International Journal of Psychology, 17, 28197.

Martínez, M., y Manoiloff, L. (2010). Evaluación neuropsicológica de la Función Ejecutiva en Adolescentes con Diferentes Patrones de Consumo de Alcohol. Revista Argentina de Ciencias del Comportamiento, 2(1), 14-23.

Martins, N y Gotuzo, A.(2015). Is it possible to promote executive functions in preschoolers? A case study in Brazil. International Journal of Child Care and Education Policy, 9(6),1 -18. doi 10.1186/s40723-0150010-2

Marino, J., Acosta, A., Zorza, J. (2011). Control ejecutivo y fluidez verbal en poblacion infantil: medidas cuantitativas, cualitativas y temporalis. Interdisciplinaria, 28(2), 245-260.

MEN (2011). Encuesta Nacional de Deserción Escolar (ENDE): Socialización de resultados principales en el marco de los encuentros regionales de construcción del Plan Sectorial de Educación. Bogotá: Encuentro Regional 2011.

Navarro Soria, I., Real Fernández, M., Lavigne Cerván, R., García-Fernández, J. M., y Piqueras Rodríguez, J. A. (2019). Predictive capacity of the Spanish neuropsychological assessment of executive functions battery when diagnosing child ADHD. Revista Latinoamericana de Psicología, 51(3), 153-161doi: http://dx.doi.org/10.14349/rlp.2019.v51.n3

Nieto-Márquez, N. L., García-Sinausía, S., y Nieto, M. Á. P. Links between motivation and metacognition and achievement in cognitive performance among primary school pupils. Anales de psicología, 37(1), 5160. https://doi.org/10.6018/analesps.383941

OECD. (2014). PISA 2012 results in focus. What 15-year-olds know and what they can do with what they know. Programme for International Student Assessment. France: Organisation for Economic Co-operation and Development.

Ortiz, T. (2013). Neurociencia y educación. Madrid: Alianza Editorial. 
Funciones Ejecutivas y Rendimiento Académico en educación primaria de la Costa Colombiana.

Peng, P., y Kievit, R. A. (2020). The development of academic achievement and cognitive abilities: A bidirectional perspective. Child Development Perspectives, 14(1), 15-20. https://doi.org/10.1111/cdep.12352

Portellano, J.A. (2005). Introducción a la neuropsicología. Madrid: Mc Graw Hill.

Portellano, Martínez-Arias y Zumárraga (2009). Evaluación Neuropsicológica de las Funciones Ejecutivas en Niños (ENFEN). Madrid: TEA Ediciones.

Puerta Morales, L. (2015). Relationship Between Cognitive Processes and Academic Performance in High School Students: Relación entre los procesos cognitivos y el rendimiento académico en estudiantes de educación básica secundaria. Psychologia. Avances de la disciplina, 9(2), 85-100.

Ramírez, M., Ostrosky-Solís, F., Fernández, A., \& Ardila-Ardila, A. (2005). Fluidez verbal semántica en hispanohablantes: un análisis comparativo. Revista de neurología, 41(8), 463-468.

Rahbari, N., y Vaillancourt1, T. (2015). Longitudinal Associations between Executive Functions and Intelligence in Preschool Children: A Multi-Method, MultiInformant Study. Canadian Journal of School Psychology. 30(4) 255-272. doi: 10.1177/0829573515594610

Reyes, S., Barreyro, J. P., \& Injoque Ricle, I. (2015). El rol de la función ejecutiva en el rendimiento académico en niños de 9 años. Revista Neuropsicología Latinoamericana. 7(2), $42-47$ DOI: $10.5579 / \mathrm{rnl} .2015 .0229$.

Roebers, C., y Jäger, K. (2014). The Relative Importance of Fine Motor Skills, Intelligence, and Executive Funtions for First Graders’ Reading and Spelling Skills. Perspectives on Language and Literacy, 40(2), 1317.

Rudasill, K.M., Gallagher, K.C. y White, J.M. (2010). Temperamental attention and activity, classroom emotional support, and academic achievement in third grade. Journal of School Psychology, 48(2), 113-34.

Scope, A., Empson, J., y McHale, S. (2010). Executive function in children with high and low attentional skills: Correspondences between behavioral and cognitive profiles. British Journal of Developmental Psychology, 28, 293-305. doi:10.1348/026151009X410371

Shaul, S., y Schwartz, M. (2014). The role of the executive functions in school readiness among preschool-age children. Reading and Writing, 27, 749-768. doi: 10.1007/s11145-013-9470-3

Stelzer, F., y Cervigni, M. (2011). Desempeño académico y funciones ejecutivas en infancia y adolescencia. Una revisión de la literatura. Revista de Investigación en Educación, 9(1), 148-156.

Suárez-Riveiro, J. M., Martínez-Vicente, M., y Valiente-Barroso, C. (2019). Rendimiento Académico según Distintos Niveles de Funcionalidad Ejecutiva y de Estrés Infantil Percibido. Educational Psychology, 26(1), 77-86. https://doi.org/10.5093/psed2019a17

Suchodoletz, A., Gestsdottir, S., Wanless, S., McClellandd, M., Birgisdottir, F., Gunzenhauser, C.,Ragnarsdottir, H. (2013).Behavioral self-regulation and relations to emergent academic skills among children in Germany and Iceland. Early Childhood Research Quarterly, 28, 62-73. doi:10.1016.2012.05.003

Tiramonti, G. (2014). Las pruebas PISA en América Latina: resultados en contexto. Avances en Supervisión Educativa, 20, 1-24

Tirapu-Ustárroz, J., y Luna-Lario, P. (2008). Neuropsicología de las funciones ejecutivas. En J. Tirapu-Ustárroz, M. Ríos-Lago y F. Maestú-Unturbe (Eds.), Manual de Neuropsicología (pp. 221-259). Barcelona: Viguera Editores. 
Titz, C., y Karbach, J. (2014). Working memory and executive functions: effects of training on academic achievement. Psychological Research, 78, 852-868. doi:10.1007/s00426-013-0537-1

Tobar, C. (2014). The influence of Sleep and Exercise, emotions and Stress, and Language on the Development of Executive Functions. Perspectives on Language and Literacy, 40(2), 19-22. doi: 10.1007/s00426013-0537-1

Toll, S., Van der Ven, S., Kroesbergen, E., y Van Luit, J. (2011). Executive Functions as Predictors of Math Learning Disabilities. Journal of Learning Disabilities, 44(6) 521-532. doi: $10.1177 / 0022219410387302$

Torres, J., Acevedo, D. y Gallo, L. (2015). Causas y consecuencias de la deserción y repitencia escolar: una visión general en el contexto Latinoamericano. Cultura Educación y Sociedad, 6(2), 157-187

Tsujimoto, S., Yamamoto, T., Kawaguchi, H., Koizumi, H., \& Sawaguchi, T. (2004). Prefrontal cortical activation associated with working memory in adults and preschool children: an event-related optical topography study. Cerebral cortex, 14(7), 703-712. DOI: 10.1093/cercor/bhh030

Valle, A., Pan, I., Núñez, J., Rosário, P., Rodríguez, S., y Regueiro, B. (2015). Deberes escolares y rendimiento académico en Educación Primaria. Anales de Psicología, 31(2), 562-569. doi: 10.6018/analesps.31.2.171131

Van der Ven, S. H., Kroesbergen, E. H., Boom, J., \& Leseman, P. P. (2013). The structure of executive functions in children: A closer examination of inhibition, shifting, and updating. British Journal of Developmental Psychology, 31(1), 70-87. DOI: 10.1111/j.2044-835X.2012.02079.x

Vandenbroucke, L., Verschueren, K., y Baeyens, D. (2017). The development of executive functioning across the transition to first grade and its predictive value for academic achievement. Learning and Instruction. 49, 103-112. Doi: https://doi.org/10.1016/j.learninstruc.2016.12.008

Wiebe, S., Sheffield, T., Mize, J., Clark, C., Chevalier, N., y Andrews, K. (2011). The structure of executive function in 3-year-olds. Journal of Experimental Child Psychology, 101, 436-452. doi:10.1016/j.jecp.2010.08.008

Yang, Y., y Raine, A. (2009). Prefrontal structural and functional brain imaging findings in antisocial, violent, and psychopathic individuals: a meta-analysis. Psychiatry Res, 174(2), 81-88

Recibido: 26-04-2020

Aceptado: 29-03-2021 